\title{
Crescimento vegetativo e teores de nutrientes em mudas de oliveira em competição com plantas daninhas
}

\author{
Larissa Madureira Martins', Maria do Céu Monteiro Cruz*, Adelson Francisco de Oliveira², \\ José Barbosa dos Santos', Miriã Cristina Pereira Fagundes' ${ }^{1}$ \\ 'Universidade Federal dos Vales do Jequitinhonha e Mucuri, Diamantina, MG, Brasil \\ ${ }^{2}$ Empresa de Pesquisa Agropecuária de Minas Gerais, Centro Tecnológigo do Sul de Minas, Lavras, MG, Brasil \\ *Autor correspondente, e-mail: mariceu@ufvjm.edu.br
}

\section{Resumo}

A oliveira tem apresentado crescimento na área plantada e na produção no Brasil. Entretanto, são poucas as informações disponíveis a respeito da interferência de plantas daninhas sobre o seu crescimento e o estado nutricional. O trabalho foi realizado com o objetivo de avaliar o crescimento vegetativo e os teores de nutrientes em mudas de oliveira em competição com diferentes espécies de plantas daninhas. Adotou-se o esquema o fatorial $2 \times 5$, sendo os fatores a combinação de duas cultivares de oliveira, 'Arbequina' e 'Ascolano', quatro espécies de plantas competidoras, Amaranthus retroflexus, Brachiaria brizantha, Bidens pilosa, Cenchrus echinatus e um tratamento testemunha (sem competição), distribuídos no delineamento inteiramente casualizado, com quatro repetições e avaliações em duas épocas: aos 45 e 90 dias após a emergência das plantas daninhas. Para cada espécie, a densidade estabelecida foi de uma planta competidora por vaso, sendo realizado o desbaste imediatamente após a emergência das espécies de plantas daninhas. O período de convivência entre as cultivares de oliveira e as plantas competidoras foi de 90 dias. A competição com as plantas daninhas interferiu no crescimento, nos teores de nutrientes e na massa seca de mudas de oliveira. A cultivar 'Ascolano' mostrou-se mais tolerante a competição com as espécies de plantas daninhas. As espécies A. retroflexus e B. pilosa apresentaram maior potencial de competição com mudas de oliveira.

Palavras-Chave: Olea europaea, competitividade, manejo, estado nutricional

\section{Vegetative growth and nutrient content in olive seedlings in competition with weeds}

\begin{abstract}
Olive tree has improved yield and production area in Brazil. However, little is known about the weeds impact on olive tree growth and nutrition. The study was carried out to evaluate the vegetative growth and nutrient contents in olive tree seedlings in competition with different weed species. The $2 \times 5$ factorial scheme was used, being the factors a combination of two olive cultivars ('Arbequina' and 'Ascolano') and four competing plant species, Brachiaria brizantha, Amaranthus retroflexus, Bidens pilosa, Cenchrus echinatus and one control treatment (without competition), distributed in a completely randomized design with four replications and the evaluations were carried out at 45 and 90 days after weed emergence. For each specie, the density of one competing plant per pot was established and the thinning was done immediately after weed emergence. The period of coexistence between olive cultivars and competing plants was 90 days after weed emergence. Weed competiton affectedolive seedlings growth, dry mass and nutrient accumulation.. 'Ascolano' was more tolerant to weed competition and A. retroflexus and B. pilosa were the species with the greater potential to competition with olive seedlings.
\end{abstract}

Keywords: Olea europaea, competitivity, management, nutritional status 


\section{Introdução}

Entre os fatores que podem afetar o desenvolvimento da oliveira, a competição com outras plantas no ambiente de cultivo é citada em função da interferência no crescimento das plantas. Visto que, a competição causada pelas plantas daninhas pode interferir significativamente na disponibilidade de nutrientes, limitando o crescimento das plantas (Elmore et al., 2004).

Entre os prejuízos causados pelas plantas daninhas, as altas quantidades de nutrientes extraídas, tem sido relatado (Ronchi et al., 2006), em decorrência da capacidade de competição destas espécies, que geralmente apresentam elevada taxa de crescimento. Assim, o entendimento da interferência causada pelas plantas daninhas sobre a oliveira se faz necessário, principalmente na fase inicial de crescimento, em virtude do potencial agressivo das espécies competidoras.

Em países em que o cultivo da oliveira já é consolidado, a competição com as plantas daninhas tem limitado significativamente a produção (Saarvedra \& Pastor, 1996), sendo a interferência relacionada à flora e da densidade dessas plantas, assim como das condições de crescimento entre e dentro de períodos de convivência (Uremis, 2005).

Entretanto, nas condições brasileiras a existência de informações relacionadas ao desenvolvimento da oliveira em competição com outras espécies ainda é incipiente. Assim, pesquisas com plantas daninhas são ferramentas úteis para determinar a importância da ocorrência de espécies competidoras e o grau de competição no sistema de produção, pois, com o surgimento das novas regiões de cultivo, essas informações permitirão a adoção de práticas de manejo adequadas para favorecer o desenvolvimento da oliveira. Entretanto, pesquisas em condições de campo não permitem avaliar com precisão os efeitos das espécies de plantas daninhas isoladamente sobre o crescimento e a absorção de nutrientes da oliveira, em virtude da interferência de diversos fatores. Assim, pesquisas realizadas com plantas em vasos permitem conhecer o comportamento das oliveiras com as espécies de plantas daninhas que comumente ocorrem no Brasil.

Desta forma, o trabalho foi realizado com o objetivo de avaliar o crescimento vegetativo e os teores de nutrientes de mudas de oliveira competindo com diferentes espécies de plantas daninhas em fase inicial de crescimento.

\section{Material e Métodos}

O trabalho foi conduzido em casa de vegetação, utilizando mudas de oliveira das cultivares 'Arbequina' e 'Ascolano' com seis meses de idade, provenientes de propagação vegetativa segundo Caballero \& Del Río (2006).

O plantio foi realizado em vasos de polietileno com capacidade para sete litros, contendo como substrato a mistura de solo e esterco na proporção de 3:1(v/v). O substrato apresentava as seguintes características químicas: $\mathrm{pH}$ (água) de 5,8; teor de matéria orgânica de 0,4 daq $\mathrm{kg}^{-1}$; P e K de 0,25 e 7,9 $\mathrm{mg} \mathrm{dm}^{-3}$, respectivamente; $\mathrm{Ca}, \mathrm{Mg}, \mathrm{Al}, \mathrm{H}+\mathrm{Al} \mathrm{e}$ CTC $_{\text {efetiva }}$ de 0,$8 ; 0,2 ; 0,04 ; 1,9$ e 1,06 $\mathrm{cmolc} \mathrm{dm}^{-3}$, respectivamente, o qual foi adubado segundo a recomendação de Mesquita et al. (2006)para a cultura da oliveira, sendo feita a adaptação para o volume do vaso.

Os tratamentos testados seguiram o esquema fatorial $2 \times 5$, sendo os fatores a combinação de duas cultivares de oliveira, 'Arbequina' e 'Ascolano', quatro espécies de plantas competidoras, Amaranthus retroflexus, Brachiaria brizantha, Bidens pilosa, Cenchrus echinatus e um tratamento testemunha (sem competição). O delineamento foi inteiramente casualizado, com quatro repetições.

Após o plantio das mudas de oliveira para os vasos e a sua aclimatação, as sementes das espécies de plantas daninhas foram semeadas diretamente nos vasos. Para cada espécie, a densidade estabelecida foi de uma planta competidora por vaso, equivalente a densidade de sete plantas por $\mathrm{m}^{2}$, sendo realizado o desbaste imediatamente após a emergência.

A diferença de crescimento em altura e em diâmetro das plantas foi determinada aos 45 e 90 dias de competição, calculada a partir da altura e do diâmetro do caule no início do experimento. 
A área foliar foi determinada ao final do período de competição, empregando-se a metodologia da massa de discos de área conhecida. Para isso foi retirada uma folha de cada planta e fazendo o uso de um furador cilíndrico retirou-se um disco foliar de área conhecida, de cada folha. Esses discos foram acondicionados em sacos de papel, assim como o restante das folhas de onde foram retirados e colocados em estufa com circulação forçada de ar a $65^{\circ} \mathrm{C}$. A área foliar (AF) foi calculada pela: massa seca das folhas $\mathrm{x}$ área dos discos/ massa seca dos discos. Com base nesses dados foi determinada a área foliar específica(AFE) pela: área foliar/massa das folhas, que relaciona a superfície da folha com a massa da própria folha.

Para determinação dos teores de nutrientes foram retiradas de 20 a 30 folhas com pecíolo por planta, retiradas no terço mediano dos ramos. As amostras foram colocadas para secar em estufa com circulação forçada de ar, a $65^{\circ} \mathrm{C}$, por aproximadamente 72 horas, até atingirem massa constante, posteriormente moídas em moinho tipo "Wiley" e submetidas à análise química. A determinação dos teores de $\mathrm{P}$ e $\mathrm{K}$ foi efetuada a partir da digestão nítrico-perclórica, obtendo-se extratos para determinação dos teores de $\mathrm{P}$ por colorimetria e K por fotometria de chama. O N total foi determinado pelo método Kjeldhal após digestão sulfúrica. Os teores de $\mathrm{Ca}, \mathrm{Mg}, \mathrm{Zn}$, Fe e $\mathrm{Mn}$ foram determinados por espectrofotometria de absorção atômica.

Os dados obtidos foram submetidos à análise de variância e comparação de médias pelo agrupamento de Scott-Knott a 5 $\%$ de probabilidade de erro para comparar o crescimento vegetativo e os teores de nutrientes das mudas de oliveira e das espécies de plantas daninhas nas condições estudadas.

\section{Resultados e Discussão}

Foi observada interação entre as espécies de plantas daninhas e as oliveiras para o crescimento em altura, área foliar e teores de $\mathrm{N}, \mathrm{K}, \mathrm{Fe}$ e Mn. Houve diferenças significativas entre as espécies de plantas daninhas para a área foliar específica e os teores de Ca, S e Zn.
Em relação ao crescimento em altura, observou-se que a presença das $\square$ espécies de plantas daninhas interferiu no crescimento das mudas, de ambas as cultivares quando comparadas às suas testemunhas. E na comparação entre as cultivares verificou-se que as mudas de 'Arbequina' competindo com B. pilosa e A. retroflexus apresentaram menor crescimento que as de 'Ascolano' (Tabela 1).

Esse comportamento evidencia que espécies de plantas avaliadas são competidoras potenciais nas condições brasileiras, pois as mudas de oliveira convivendo com plantas daninhas apresentaram menor crescimento, em decorrência da menor disponibilidade de nutrientes causada pela competição com as plantas daninhas, conforme observado com as espécies de plantas daninhas de outros países produtores (Elmoreet al., 2004).

Comparando-se o crescimento nas duas épocas avaliadas, observa-se que aos 45 dias de convivência não houve diferença no crescimento das mudas, enquanto aos 90 dias, as mudas cultivadas sem a presença de plantas daninhas apresentaram maior crescimento (Tabela 1). Esse comportamento sugere que - tempo de convivência é determinante para ocorrer a interferência resultante da competição das espécies daninhas sobre as mudas de oliveiras estudadas, possivelmente pelo esgotamento mais rápido dos recursos do meio de cultivo, considerando a elevada taxa de crescimento das espécies competidoras comparadas as mudas de oliveira.

Para o crescimento do diâmetro do caule não foi constatada a interferência das plantas daninhas nas mudas de ambas as cultivares. E comparando-se as cultivares, observou-se que a 'Arbequina' apresentou menor crescimento quando em competição B. pilosa e A. retroflexus e também no tratamento testemunha (Tabela 1). O resultado observado em relação ao crescimento das plantas sugere que as espécies interferem de maneira diferenciada e que as cultivares de oliveira apresentam diferenças em relação à capacidade competitiva.

Em relação à área foliar, verificou-se que a cultivar 'Arbequina' apresentou menor crescimento quando estava em competição 
com as espécies de plantas daninhas. Já a 'Ascolano' mostrou-se tolerante competindo com B. brizantha e C. echinathus, sobressaindose à sua testemunha e a 'Arbequina' convivendo com estas espécies, evidenciando a sua menor susceptibilidade aos efeitos de competição. Para a área foliar específica, que relaciona a área foliar com a massa da folha, nas mudas da cultivar 'Arbequina' em competição com as plantas daninhas observou-se que as folhas de menor tamanho apresentaram maior espessura, enquanto a 'Ascolano' não diferiu das plantas do tratamento testemunha (Tabela 2).

Tabela 1. Diferença de crescimento em altura e diâmetro nas mudas de oliveira (Olea europaea L.), 'Arbequina' e 'Ascolano', aos 45 e 90 dias de competição com plantas daninhas.

\begin{tabular}{|c|c|c|c|c|}
\hline \multirow{3}{*}{ Espécies de plantas daninhas } & \multicolumn{4}{|c|}{ Altura $(\mathrm{cm})^{*}$} \\
\hline & \multicolumn{2}{|c|}{ Cultivares } & \multicolumn{2}{|c|}{ Época } \\
\hline & 'Arbequina' & 'Ascolano' & 45 dias & 90 dias \\
\hline Testemunha & $6,87 \mathrm{Aa}$ & $5,50 \mathrm{Ab}$ & $4,50 \mathrm{~A}$ & $7,87 \mathrm{~A}$ \\
\hline Bidens pilosa & $2,62 \mathrm{Bb}$ & $3,19 \mathrm{Ba}$ & $2,56 \mathrm{~A}$ & $3,25 B$ \\
\hline Amaranthus retroflexus & $2,06 \mathrm{Bb}$ & $3,62 \mathrm{Ba}$ & $3,62 \mathrm{~A}$ & $2,06 \mathrm{~B}$ \\
\hline Cenchrus echinatus & $3,50 \mathrm{Ba}$ & $3,06 \mathrm{Ba}$ & $2,19 \mathrm{~A}$ & $4,37 \mathrm{~B}$ \\
\hline Brachiaria brizantha & $4,02 \mathrm{Ba}$ & $3,00 \mathrm{Ba}$ & $3,87 \mathrm{~A}$ & $3,15 \mathrm{~B}$ \\
\hline CV $(\%)^{1}$ & \multicolumn{2}{|c|}{22,6} & \multicolumn{2}{|c|}{26,4} \\
\hline Espécies de plantas daninhas & \multicolumn{4}{|c|}{ Diâmetro (mm)* } \\
\hline Testemunha & $0,35 \mathrm{Ab}$ & $0,98 \mathrm{Aa}$ & $0,75 \mathrm{~A}$ & $0,58 \mathrm{~A}$ \\
\hline Bidens pilosa & $0,58 \mathrm{Ab}$ & $1,00 \mathrm{Aa}$ & $0,84 \mathrm{~A}$ & $0,74 \mathrm{~A}$ \\
\hline Amaranthus retroflexus & $0,44 \mathrm{Ab}$ & $0,76 \mathrm{Aa}$ & $0,73 \mathrm{~A}$ & $0,74 \mathrm{~A}$ \\
\hline Cenchrus echinatus & $0,47 \mathrm{Aa}$ & $0,82 \mathrm{Aa}$ & $0,55 \mathrm{~A}$ & $0,46 \mathrm{~A}$ \\
\hline Brachiaria brizantha & $0,67 \mathrm{Aa}$ & $0,93 \mathrm{Aa}$ & $0,76 \mathrm{~A}$ & $0,85 \mathrm{~A}$ \\
\hline $\mathrm{CV}(\%)^{1}$ & \multicolumn{2}{|c|}{27,4} & \multicolumn{2}{|c|}{21,8} \\
\hline
\end{tabular}

Tabela 2. Área foliar e área foliar específica diâmetro nas mudas de oliveira (Olea europaea L.), 'Arbequina' e 'Ascolano', aos 90 dias de competição.

\begin{tabular}{lcccc}
\hline \multirow{2}{*}{ Espécies de plantas daninhas } & \multicolumn{2}{c}{ Área foliar $\left(\mathrm{cm}^{2}\right)$} & \multicolumn{2}{c}{ Área foliar específica $\left(\mathrm{cm}^{2} \mathrm{~g}^{-1}\right)$} \\
\cline { 2 - 5 } & 'Arbequina' & 'Ascolano' & 'Arbequina' & 'Ascolano' \\
\hline Testemunha & $6,06 \mathrm{Aa}$ & $5,71 \mathrm{Ba}$ & $57,6 \mathrm{Aa}$ & $54,0 \mathrm{Aa}$ \\
Bidens pilosa & $5,52 \mathrm{Ba}$ & $5,81 \mathrm{Ba}$ & $44,5 \mathrm{Ba}$ & $47,6 \mathrm{Aa}$ \\
Amaranthus retroflexus & $5,12 \mathrm{Ba}$ & $5,84 \mathrm{Ba}$ & $44,3 \mathrm{Bb}$ & $53,1 \mathrm{Aa}$ \\
Cenchrus echinatus & $4,84 \mathrm{Bb}$ & $6,37 \mathrm{Aa}$ & $45,9 \mathrm{Ba}$ & $49,5 \mathrm{Aa}$ \\
Brachiaria brizantha & $5,12 \mathrm{Bb}$ & $6,93 \mathrm{Aa}$ & $44,4 \mathrm{Ba}$ & $49,7 \mathrm{Aa}$ \\
\hline \multicolumn{1}{c}{ CV (\%)' } & \multicolumn{3}{c}{5,33} \\
\hline
\end{tabular}

*Médias seguidas por letras maiúsculas diferentes na coluna diferem entre si pelo agrupamento de Scott-Knott e seguidas de letras minúsculas diferentes na linha diferem entre si pelo teste F, a $5 \%$ de probabilidade de erro. 'Coeficiente de variação.

Estes resultados sugerem que as cultivares de oliveira apresentam capacidade diferenciada de competição com plantas daninhas e que a competição pode ocasionar prejuízos ao crescimento das mudas, caso não seja efetuado o manejo, pois além da menor disponibilidade de nutrientes, a limitação hídrica em condições de competição, como a observada no presente estudo, interfere noíndice de área foliar, que resulta em menor capacidade fotossintética (Scalon et al., 2011). A diferença em relação às mudas da 'Ascolano' pode ser atribuída ao seu hábito de crescimento ereto, o que contribui para diminuir o sombreamento da sua área foliar, favorecendo maior crescimento em comparação as mudas da cultivar 'Arbequina', que devido à arquiteturade copa aberta foram mais afetadas pela competição das plantas daninhas.

Com relação aos teores de N (Tabela 3), observou-se que as plantas da cultivar Arbequina apresentaram menores teores de $\mathrm{N}$ quando estavam em competição com B. pilosa e C. echinatus, já na 'Ascolano' os menores teores de $\mathrm{N}$ foram verificados com $\mathrm{A}$. retroflexus e B. brizantha. A redução desse nutriente compromete o crescimento vegetativo da oliveira, principalmente na fase inicial, após o 
plantio das mudas no campo, visto que grande quantidadedo nitrogênio é utilizada para o crescimento vegetativo, além de favorecer a redução da alternância de produção e aumento da porcentagem de flores perfeitas e consequente produtividade da oliveira em plantas adultas (Chouliaras et al., 2009).

Tabela 3. Teores de nitrogênio (N), fósforo (P), potássio (K), Cálcio (Ca), Magnésio (Mg) e enxofre (S) em folhas de oliveira (Oleae uropaea L.) 'Arbequina' (ARB) e 'Ascolano' (ASC) cultivadas em vaso, aos 90 dias de competição com plantas daninhas.

\begin{tabular}{|c|c|c|c|c|c|c|}
\hline \multirow{3}{*}{ Plantas daninhas } & \multicolumn{2}{|c|}{$\mathrm{N}$} & \multicolumn{2}{|c|}{$P$} & \multicolumn{2}{|c|}{ K } \\
\hline & \multicolumn{4}{|c|}{ g kg ${ }^{-1}$--- } & --------- & \\
\hline & ARB & ASC & ARB & ASC & ARB & ASC \\
\hline Testemunha & $33,6 \mathrm{Aa}$ & $25,6 \mathrm{Ab}$ & $1,64 \mathrm{Aa}$ & $1,18 \mathrm{Ab}$ & $35,8 \mathrm{Aa}$ & $39,4 \mathrm{Aa}$ \\
\hline Bidens pilosa & $31,9 \mathrm{Ba}$ & $26,1 \mathrm{Ab}$ & $1,69 \mathrm{Aa}$ & $0,99 \mathrm{Ab}$ & $37,8 \mathrm{Aa}$ & $33,4 \mathrm{Ba}$ \\
\hline Amaranthus retroflexus & $32,9 \mathrm{Aa}$ & $23,5 \mathrm{Bb}$ & $1,80 \mathrm{Aa}$ & $1,02 \mathrm{Ab}$ & $38,8 \mathrm{Aa}$ & $34,4 \mathrm{Ba}$ \\
\hline Cenchrus echinatus & $32,5 \mathrm{Ba}$ & $26,1 \mathrm{Ab}$ & $1,69 \mathrm{Aa}$ & $0,97 \mathrm{Ab}$ & $31,4 \mathrm{Ba}$ & $33,5 \mathrm{Ba}$ \\
\hline Brachiaria brizantha & $33,2 \mathrm{Aa}$ & $23,6 \mathrm{Bb}$ & $1,92 \mathrm{Aa}$ & $0,95 \mathrm{Ab}$ & $34,2 \mathrm{Ba}$ & $34,9 \mathrm{Ba}$ \\
\hline \multirow[t]{3}{*}{$\mathrm{CV}(\%)^{1}$} & \multicolumn{2}{|c|}{3,72} & \multicolumn{2}{|c|}{19,7} & \multicolumn{2}{|c|}{9,07} \\
\hline & \multicolumn{2}{|c|}{$\mathrm{Ca}$} & \multicolumn{2}{|c|}{$M g$} & \multicolumn{2}{|c|}{$S$} \\
\hline & \multicolumn{6}{|c|}{------------------------ $\mathrm{g} \mathrm{kg}^{-1}$---------------------- } \\
\hline Testemunha & $13,5 \mathrm{Aa}$ & $17,5 \mathrm{Aa}$ & 1,90 Aa & $2,20 \mathrm{Aa}$ & $2,06 \mathrm{Aa}$ & $1,70 \mathrm{Aa}$ \\
\hline Bidens pilosa & $9,2 \mathrm{Ba}$ & $9,9 \mathrm{Ba}$ & $1,76 \mathrm{Aa}$ & $2,18 \mathrm{Aa}$ & $2,03 \mathrm{Aa}$ & $1,54 \mathrm{Ba}$ \\
\hline Amaranthus retroflexus & $12,6 \mathrm{Aa}$ & $13,2 \mathrm{Ba}$ & $2,06 \mathrm{Aa}$ & $2,21 \mathrm{Aa}$ & $2,46 \mathrm{Aa}$ & $1,67 \mathrm{Ab}$ \\
\hline Cenchrus echinatus & $5,9 \mathrm{Bb}$ & $11,6 \mathrm{Ba}$ & $1,13 \mathrm{Bb}$ & $2,15 \mathrm{Aa}$ & $1,69 \mathrm{Ba}$ & $1,41 \mathrm{Ba}$ \\
\hline Brachiaria brizantha & $11,8 \mathrm{Aa}$ & $12,3 \mathrm{Ba}$ & $1,75 \mathrm{Ab}$ & $2,44 \mathrm{Aa}$ & $2,03 \mathrm{Aa}$ & $1,11 \mathrm{Bb}$ \\
\hline $\mathrm{CV}(\%)^{1}$ & \multicolumn{2}{|c|}{22,1} & \multicolumn{2}{|c|}{16,7} & \multicolumn{2}{|c|}{20,1} \\
\hline
\end{tabular}

Dessa forma, é essencial realizaro manejo adequado da cultura desde a fase inicial, pois a interferência na absorção de minerais limita o processo fotossintético, além disso, é na fase inicial que as plantas daninhas exercem a maior agressividade na competição por nutrientes, quando o desenvolvimento das raízes da oliveira é limitado e as plantas daninhas alcançam porte equivalente à cultura, com maior taxa de crescimento e, consequentemente, maior velocidade de extração de nutrientes do solo.

Quanto ao P (Tabela 3), as mudas não apresentaram diferenças significativas em relação à testemunha. O resultado observado pode ter ocorrido em função da pouca mobilidade desse nutriente no solo. Além disso, - sistema radicular da oliveira possui uma alta capacidade de absorção pelo seu elevado número de raízes finas.

Para o K (Tabela 3), as mudas da cultivar'Arbequina' que conviveram com $C$. echinatuse $B$.brizantha apresentaram teores menores que a testemunha. Enquanto para a cultivar 'Ascolano' os menores teores foram observados nas mudas em competição com as espécies de plantas daninhas. Comportamento semelhante foi observado no cafeeiro com a redução dos teores de potássio em decorrência da competição com plantas daninhas (Ronchi et al., 2006). A escassez de nutrientes ocasiona o desequilíbrio entre o conteúdo mineral e o incremento de matéria seca, interferindo no desenvolvimento da espécie, pois na fase inicial, a disponibilidade de $K$ é importante para a oliveira devido ao seu papel no uso eficiente da água (Restrepo-Diaz et al., 2008), o que pode ser prejudicado sob competição com outras plantas.

Com relação aos teores de $\mathrm{Ca}, \mathrm{Mg}$ e $S$ (Tabela 4), as mudas da cultivar Arbequina apresentaram menores teores quando estava em convivência com B. pilosa e C. echinatus e para a cultivar 'Ascolano' os menores teores de $S$ foram observados nas mudas que competiram com B. pilosa, C. echinatus e B.brizantha e de Ca com todas as espécies competidoras. Entre os nutrientes que sofreram interferência pela competição das plantas daninhas, os teores de Ca nas mudas competindo com B. pilosa e C. echinatus estavam abaixo do nível considerado 
bom para oliveira. Isso pode ter ocorrido em função da mobilidade do elemento no floema, apresentando acúmulo mínimo nas folhas durante o período de crescimento vegetativo (Chatzistathis et al., 2011).

A capacidade competitiva dessas espécies daninhas já foi constatada em diversas espécies agrícolas anuais e perenes nas condições brasileiras. B. pilosa competindo com as culturas da soja, feijão (Procópio et al., 2005) e cafeeiro (Ronchiet al., 2006) promoveu elevada extração de nutrientes do solo, devido ao alto volume de solo explorado pelo sistema radicular e $A$. retroflexus em competição com outras espécies causou redução da produção de massa seca e da produtividade, devido ao seu grande porte e por apresentar alta capacidade competitiva (Bukun,2011).

Na avaliação do potencial competitivo entre as cultivares, observou-se que para todos os nutrientes a 'Arbequina' apresentou maiores teores dos nutrientes em relação à 'Ascolano'. Essa diferença pode ser atribuída ao hábito de crescimento da cultivar, pois as mudas da cultivar 'Ascolano' apresentaram maior crescimento vegetativo, possivelmente utilizaram maiores quantidades de nutrientes. Variações nos teores de nutrientes em função do crescimento já foi constatada em oliveiras da cultivar 'Nabali' que apresentaram maiores teores de N, P e K, devido ao seu crescimento lento, quando comparada com outras cultivares (Freihat \& Masa'Deh, 2006).

Para os micronutrientes, foi observado menores teores de Fe, Mn e Zn nas mudas de ambas as cultivares de oliveira em competição com as diferentes espécies de plantas daninhas (Tabela 4). Emrelação ao B, não foram observadas diferenças significativas. Esse comportamento ocorreu, provavelmente, porque o B é um elemento utilizado principalmente para a formação de gemas florais e desenvolvimento dos frutos, época em que o conteúdo foliar do nutriente sofre redução, em decorrência da alta quantidade que é mobilizada para a formação das flores e frutos (Carvalho et al., 2013).

Tabela 4. Teores de boro (B), ferro (Fe), manganês (Mn) e zinco (Zn) em folhas de oliveira (Olea europaea L.), 'Arbequina' e 'Ascolano', aos 90 dias de competição com plantas daninhas.

\begin{tabular}{|c|c|c|c|c|}
\hline \multirow{3}{*}{ Plantas daninhas } & \multicolumn{2}{|l|}{ B } & \multicolumn{2}{|c|}{$\mathrm{F}$} \\
\hline & \multicolumn{4}{|c|}{ - } \\
\hline & 'Arbequina' & 'Ascolano' & 'Arbequina' & 'Ascolano' \\
\hline Testemunha & $19,4 \mathrm{Aa}$ & $18,9 \mathrm{Aa}$ & $152,8 \mathrm{Aa}$ & $105,3 \mathrm{Ab}$ \\
\hline Bidens pilosa & $23,4 \mathrm{Aa}$ & $16,8 \mathrm{Ab}$ & $125,4 \mathrm{Ba}$ & $119,3 \mathrm{Aa}$ \\
\hline Amaranthus retroflexus & $22,1 \mathrm{Aa}$ & $19,9 \mathrm{Aa}$ & $129,6 \mathrm{Ba}$ & $94,7 \mathrm{Bb}$ \\
\hline Cenchrus echinatus & $21,5 \mathrm{Aa}$ & $18,3 \mathrm{Aa}$ & $126,4 \mathrm{Ba}$ & $89,2 \mathrm{Bb}$ \\
\hline Brachiaria brizantha & $20,7 \mathrm{Aa}$ & $17,4 \mathrm{Aa}$ & $138,3 \mathrm{Ba}$ & $105,7 \mathrm{Ab}$ \\
\hline \multirow[t]{3}{*}{$\mathrm{CV}(\%)^{1}$} & \multicolumn{2}{|c|}{18,6} & \multicolumn{2}{|c|}{11,8} \\
\hline & \multicolumn{2}{|c|}{$M n$} & \multicolumn{2}{|c|}{$\mathrm{Zn}$} \\
\hline & \multicolumn{4}{|c|}{ - mg kg ${ }^{-1}$ - } \\
\hline Testemunha & $28,5 \mathrm{Ab}$ & $54,6 \mathrm{Aa}$ & $27,2 \mathrm{Aa}$ & $28,3 \mathrm{Aa}$ \\
\hline Bidens pilosa & $27,1 \mathrm{Aa}$ & $23,9 \mathrm{Ca}$ & $24,7 \mathrm{Ba}$ & $21,7 \mathrm{Ba}$ \\
\hline Amaranthus retroflexus & $26,5 \mathrm{Aa}$ & $32,2 \mathrm{Ba}$ & $23, \mathrm{OBa}$ & $19, \mathrm{OBa}$ \\
\hline Cenchrus echinatus & $23,4 \mathrm{Ba}$ & $24,9 \mathrm{Ca}$ & $22,5 \mathrm{Ba}$ & $20,7 \mathrm{Ba}$ \\
\hline Brachiaria brizantha & $24,4 \mathrm{Ba}$ & $31,7 \mathrm{Bb}$ & $23,5 \mathrm{Ba}$ & $19,5 \mathrm{Ba}$ \\
\hline $\mathrm{CV}(\%)^{\prime}$ & \multicolumn{2}{|c|}{18,1} & \multicolumn{2}{|c|}{10,0} \\
\hline
\end{tabular}

*Médias seguidas por letras maiúsculas diferentes na coluna diferem entre si pelo agrupamento de Scott-Knott e seguidas de letras minúsculas diferentes na linha diferem entre si pelo teste F, a 5\% de probabilidade de erro. 'Coeficiente de variação.

Para o nutriente Fe, as mudas da cultivar 'Arbequina' apresentaram menores teores em competição com todas as plantas daninhas, enquanto para a 'Ascolano' os menores teores foram observados nas mudas que competiram com A. retroflexus e C. echinatus. Para o $\mathrm{Mn}$, os menores teores nas mudas da cultivar 'Arbequina' foram verificados em convivência com C. echinatus e B. brizantha e as de 'Ascolano' em competição com as espécies de plantas daninhas. Para o $\mathrm{Zn}$ o resultado foi semelhante, observando-se os menores teores nas mudas de ambas as cultivares de oliveira em convivência com as espécies de plantas daninhas (Tabela 4).

Nesse estudo, o crescimento das 
plantas competidoras se deu mais rápido que o das mudas de oliveira. Gustafson et al. (2004) afirmam que a elevada velocidade de emergência e de crescimento inicial das plantas daninhas as tornam prioritárias na utilização dos recursos do meio e, por isso, geralmente estas levam vantagem na utilização em situações de concorrência. Diante dos resultados obtidos com esse estudo, observou-se a necessidade de realizar o manejo correto das plantas daninhas nos olivais, principalmente na fase inicial de crescimento.

\section{Conclusões}

A competição com as plantas daninhas interferiu no crescimento e nos teores de nutrientes de mudas de oliveira, sendo a cultivar 'Ascolano' mais tolerante a competição com as espécies de plantas daninhas.

As espécies A. retroflexus e B. pilosa foram as que apresentaram maior potencial de competição com as mudas de oliveira.

\section{Agradecimentos}

A Empresa de Pesquisa Agropecuária de Minas Gerais - EPAMIG pela doação das mudas de oliveiras utilizadas nesta pesquisa. À Fundação de Amparo à Pesquisa de Minas Gerais (FAPEMIG) e ao Conselho Nacional de Desenvolvimento Científico e Tecnológico (CNPq) e à Coordenadoria de Aperfeiçoamento do Pessoal de Nível Superior (CAPES) pela concessão de bolsas de estudo aos discentes envolvidos.

\section{Referências}

Bukun, B. 2011. Influence of redroot pigweed (Amaranthus retroflexus) density and biomass on peanut (Arachis hypogaea) yield. African Journal of Biotechnology 10: 19542-19546.

Caballero, J. M., Del Río, C. 2006. Propagação da oliveira por enraizamento de estacas semilenhosas sob nebulização. Informe Agropecuário 27:33-46.

Carvalho, R. P., Cruz, M. C. M., Oliveira, A. F.,Fagundes, M. C.P. 2013. Teores de nutrientes de dois cultivares de oliveira durante o crescimento vegetativo e o florescimento. Revista Ceres 60:569-576.

Chatzistathis, T. A., Papadakis, I. E., Therios, I. N., Giannakoula, A., Dimassi, K. 2011 .ls chlorophyll fluorescence technique a useful tool to assess manganese deficiency and toxicity stress in olive plants? Journal of Plant Nutrition 34: 98-1 14.

Chouliaras, V., Tasioula, M., Chatzissavvidis, C., Therios, I., Tsabolatidou, E. 2009.The effects of a seaweed extract in addition to nitrogen and boron fertilization on productivity, fruit maturation, leaf nutritional status and oil quality of the olive (Olea europaea L.) cultivar Koroneiki. Journal of the Science of Food and Agriculture 89:984-988.

Elmore, C.L. Cudney, D. W., Donaldson, D. R. 2004. Olive: Integrated Weed Management. In: Ohlendorf, B.M., Flint, L., Brush, M., King, S. (Eds.). UC IPM Pest Management Guidelines: Olive. UC ANR Publication 3452. University of California, p. 27-38.

Freihat, N.M., Masa'deh, Y.K. 2006. Response of two-year-old trees of four Olive cultivars to fertilization. American-Eurasian Journal Agriculture \& Environmental Science 1:185-190.

Gustafson, D.J., Gibson, D. J., Nickrent, D. L. 2004. Competitive relationships of Andropogon gerardii (big bluestem) from remnant and restored native populations and select cultivated varieties. Functional Ecology 18:451-457.

Mesquita, H. A.,Fráguas, J. C., Paula, M. B. 2006. Adubação e nutrição de oliveira. Informe Agropecuário 27:68-72.

Procópio, S.O., Santos, J. B., Pires, F. R., Silva, A. A., Mendonça, E. S. 2005. Absorção e utilização do fósforo pelas culturas da soja e do feijão e por plantas daninhas. Revista Brasileira de Ciência do Solo 29:911-921.

Restrepo-Diaz, H.,Benlloch, M., Navarro, C., Fernández-Escobar, R. 2008. Potassium fertilization of rain fed olive orchards. Scientia Horticulturae $116: 399-403$.

Ronchi, C. P., Silva, A. A. 2006. Effects of weed species competition on the growth of young coffee plants. Planta Daninha 24:415-423.

Saaverdra, M., Pastor, M. 1996. Weed populations in olive groves under non-tillage and conditions of rapid degradation of simazine. Weed Research 36:1-14.

Scalon, S. P. Q., Mussury, R. M., Euzébio, V. L. M., Kodama, F. M., Kissmann, C. 2011 . Estresse hídrico no metabolismo e crescimento inicial de mudas de mutambo (Guazuma ulmifolia Lam.). Ciência Florestal 21:655-662.

Uremis, I. 2005.Determination of weed species and their frequency and density in olive groves in Hatay province of Turkey. Pakistan Journal Biological Sciences 8:164-167. 